\title{
Modeling Hydration Status Given Daily Measures of Body Mass, Urine Color, and Thirst
}

\author{
Travis Anderson William M. Adams Laurie Wideman \\ Department of Kinesiology, University of North Carolina at Greensboro, Greensboro, NC, USA
}

\section{Keywords}

Machine learning $\cdot$ Copeptin · Vasopressin $\cdot$ Hydration monitoring $\cdot$ Health

\begin{abstract}
Background: The ability to monitor changes in daily hydration status is critical for human health and performance. Monitoring changes in body weight (W), urine color (U), and thirst $(\mathrm{T})$ was proposed as a simple, low-cost method for daily hydration monitoring [1]. However, the ability of these metrics to accurately predict 24-h hydration status is yet to be fully tested. Objective: The purpose of this study was to assess the degree to which daily monitoring of $\mathrm{W}, \mathrm{U}$, and $\mathrm{T}$ (i.e., the WUT model) could accurately predict 24-h hydration status. Methods: Thirty-five male and female adults (age: $23.4 \pm 4.1$ years, height: $173.0 \pm 10.3 \mathrm{~cm}$, mass: $77.2 \pm 18.2 \mathrm{~kg}$, body fat: $18.4 \pm 8.4 \%$ ) were monitored for 8 consecutive days. Assessments on each morning included a 24-h urine sample for urine osmolality (UOSM24), a first void spot urine for urine color $(U)$, nude body weight $(W)$, and perceived thirst (T) using a 1-9 Likert scale. On days 7 and 8, a blood sample was taken for copeptin assessment [2]. If UOSM24 was $>800 \mathrm{mOsm} \cdot \mathrm{kg}^{-1}$ on any day, the participants were classified as hypohydrated. Multiple research questions were explored. First, models tested the degree to which $\mathrm{W}, \mathrm{U}$, and $\mathrm{T}$
\end{abstract}

karger@karger.com www.karger.com/anm

Karger $\frac{1}{6}$
(C) 2022 The Author(s)

Published by S. Karger AG, Basel

This article is licensed under the Creative Commons Attribution 4.0 International License (CC BY) (http://www.karger.com/Services/ OpenAccessLicense). Usage, derivative works and distribution are permitted provided that proper credit is given to the author and the original publisher. could predict UOSM24 (RQ1). Classification models assessed the ability to predict whether an individual was hypohydrated (UOSM24 >800 mOsm $\cdot \mathrm{kg}^{-1}$; RQ2). Last, models tested the degree to which $\mathrm{W}, \mathrm{U}$, and $\mathrm{T}$ could predict concentrations of copeptin (RQ3). For each question, 4 separate modeling approaches were used: linear regression (LR), elastic net regression (EN), extreme gradient boosted random forests (XB), and a single hidden layer neural network (NN). Eighty percent of the data (RQ1/RQ2 $n=207, \mathrm{RQ} 3 n=49)$ were used to train the models, while $20 \%$ of the data were held out (RQ1/ $\mathrm{RQ} 2 n=51, \mathrm{RQ} 3 n=10)$ for model validation. Within the training data, bootstrap samples (XB) and cross-fold validation (EN and NN) samples were used to optimize model hyperparameters. Prediction accuracy for regression analyses were assessed via $R^{2}$ and root mean square error (RMSE), and for classification analyses via area under the curve of the receiver operating characteristic. Results: The results demonstrated that the NN model performed best when predicting UOSM24 $\left(R^{2}=0.532, \mathrm{RMSE}=228 \mathrm{mOsm} \cdot \mathrm{kg}^{-1}\right)$, but all models had relatively poor fit and large errors during validation. All classification models were moderately effective at discriminating between the binary hydration status of individuals (area under the curve of the receiver operating characteristic range: $0.661-0.696$ ). Linear regression (Fig. $1 ; R^{2}=0.929$, $\left.\mathrm{RMSE}=1.49 \mathrm{pmol} \cdot \mathrm{L}^{-1}\right), \mathrm{XB}\left(R^{2}=0.834, \mathrm{RMSE}=3.11 \mathrm{pmol} \cdot \mathrm{L}^{-1}\right)$, $\mathrm{EN}\left(R^{2}=0.890\right.$, RMSE $\left.=2.13 \mathrm{pmol} \cdot \mathrm{L}^{-1}\right)$, and NN $\left(R^{2}=0.930\right.$, 
RMSE $=1.84 \mathrm{pmol} \cdot \mathrm{L}^{-1}$ ) were able to predict copeptin concentrations with relatively low error. Conclusions: The WUT model accurately predicts copeptin concentrations on out of training data observations, suggesting that first morning measures of $\mathrm{W}, \mathrm{U}$, and $\mathrm{T}$ are effective for tracking hydration

Fig. 1. Predicted copeptin from the LR model compared to observed copeptin in the out of training sample observations. The solid gray line represents perfect prediction. LR, linear regression.

\section{Statement of Ethics}

All subjects gave their written informed consent, and this study protocol was reviewed and approved by the Institutional Review Board at the University of North Carolina at Greensboro (IRB \#180063).

\section{Conflict of Interest Statement}

Travis Anderson has received speakers fees from Danone Research.

\section{Funding Sources}

This research was supported by a School of Health and Human Sciences faculty research grant at the University of North Carolina at Greensboro.

\section{Author Contributions}

Travis Anderson contributed to data collection, conceptualization, data analysis, primary author, critical review, and editing. William M. Adams is the principal investigator and contributed to status by indirectly monitoring arginine vasopressin. More research is needed to determine potential cut-scores of predicted copeptin levels to aid practitioner decisions.

(c) 2022 The Author(s)

Published by S. Karger AG, Basel

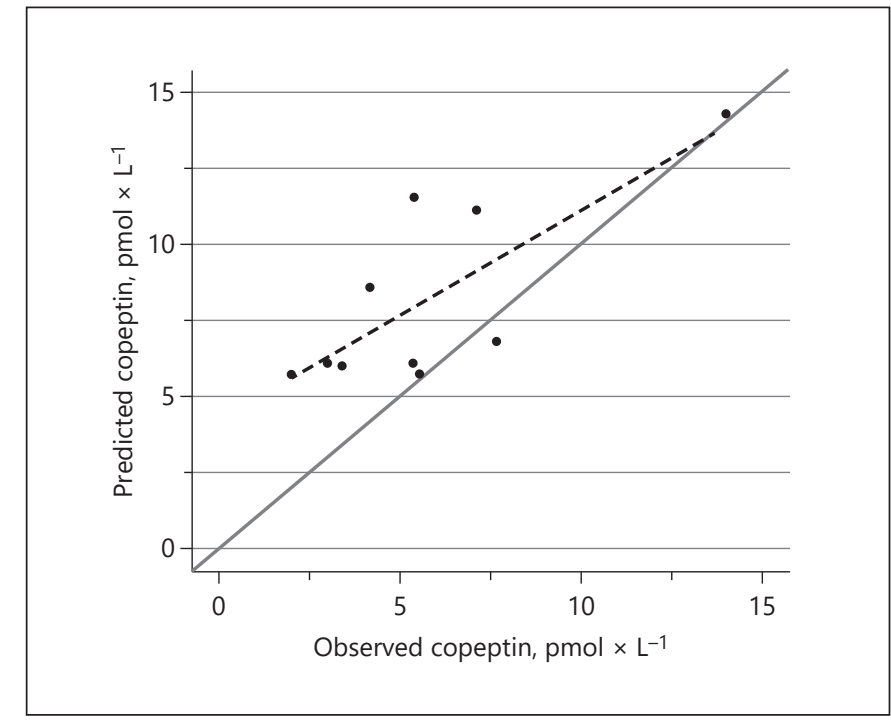

data collection, conceptualization, critical review, and editing. Laurie Wideman is the senior investigator and contributed to conceptualization, critical review, and editing.

\section{Data Availability Statement}

The data that support the findings of this study are not publicly available due to ongoing analysis but are available from T.A. (t_ander2@uncg.edu) upon reasonable request.

\section{References}

1 Cheuvront SN, Sawka MN. Hydration assessment of athletes. Sports Sci Exchange. 2005;18(2):1-6.

2 Morgenthaler NG, Struck J, Alonso C, Bergmann A. Assay for the measurement of copeptin, a stable peptide derived from the precursor of vasopressin. Clin Chem. 2006;52(1):112-9. 